\title{
ESPACIO, TERRITORIO Y LA POLÍTICA DEL AGUA EN LAS SIERRAS CHICAS DE CÓRDOBA
} - ARGENTINA

\section{Space, territory and the politics of water in Sierras Chicas - Córdoba - Argentina.}

\author{
Adrian Koberwein \\ Universidad de Buenos Aires - CONICET, Buenos Aires, Argentina \\ adriankoberwein@gmail.com
}

Artigo recebido em 15/05/2014 e aceito para publicação em 31/05/2015

RESUMEN: Diversas iniciativas llevadas adelante por grupos e instituciones para encontrar soluciones a la crisis hídrica que sufre la zona de sierras chicas de la Provincia de Córdoba en la Argentina, se articulan con ciertas dinámicas espacio-territoriales. Basándome en un análisis etnográfico y comparativo, en este artículo describo y analizo diferentes conflictos alrededor de la apropiación del recurso hídrico. Considero que estos conflictos sólo pueden explicarse en relación a su dimensión espacio-territorial. Entiendo al espacio-territorio en términos de su producción social. Es decir, en términos del cambio y la transformación producto de la actividad humana. Propongo que, para entender al espacio-territorio en términos procesuales y relacionales, específicamente en términos de su relación con la apropiación de los recursos hídricos, es necesario atender asimismo a las formas fijas y relativamente permanentes que éste adopta, y comprender la manera específica en que las dinámicas de apropiación y control de recursos naturales como el agua se articulan con las diferentes formas en que el espacio-territorio es producido. Palabras clave: Recursos Hídricos. Conflictos. Espacio-Territorio

ABSTRACT: A diversity of initiatives carried out by groups and institutions to find solutions to the water crisis affecting the area of 'Sierras Chicas' in the Province of Córdoba, Argentina, are articulated with certain spaceterritorial dynamics. In this article, and based on an ethnographic and comparative analysis, I describe and analyze different conflicts about the appropriation of water resources. These conflicts can only be explained in relation to their spatial and territorial dimension. I understand space-territory in terms of its social production; that is, in terms of change and transformation that are product of human activity. To understand space-territory in processual and relational terms, specifically in relation to the appropriation of water resources, I propose that we must also attend the relatively permanent and fixed forms that it adopts, and understand the specific ways in which the dynamics of appropriation and control of natural resources such as water, are articulated with different forms of space-territory production.

Keywords: Water Resources. Conflicts. Space-Territory. 


\section{INTRODUCCIÓN}

El presente artículo se enmarca en un análisis antropológico más amplio desde el cual abordo la relación entre recursos naturales, específicamente recursos hídricos, y el espacio-territorio en las Sierras Chicas de la provincia de Córdoba, Argentina. Se trata de un análisis encarado desde la antropología social; por lo tanto, el énfasis está puesto en el aspecto social de esta relación. Aquí propongo tratar con la manera en que una diversidad de actores involucrados en la 'política de los recursos hídricos' en la zona mencionada, despliegan prácticas que inciden sobre la organización espacio-territorial.

Con el término 'política de recursos hídricos' me refiero al conjunto de actores, relaciones, acciones y procesos sociales que influyen en la dinámica de la captación, preservación, distribución, uso, control y apropiación de los recursos hídricos en general. Descarto, de esta manera, todo uso restringido del término, ya sea como sinónimo de gestión de los recursos hídricos, o como reducido a los términos de una política específica sobre el tema, fuera estatal o privada. La pregunta que me guía en este trabajo es: ¿de qué manera las acciones de diferentes actores en torno al problema del agua intervienen en la producción social del espacio-territorio? Utilizo el término 'espacio-territorio' para connotar tanto los elementos dinámicos como los relativamente estables que son producto de la producción social del espacio.
Algunos resultados de esta producción son formas fijas y fijadas con intenciones permanentes. Tal es el caso de los territorios propiamente dichos. Es decir, de las jurisdicciones con límites socialmente establecidos que son producto de una planificación calculada y que permiten, por esa misma razón, que sean asimismo objeto de planificación y cálculo (HARVEY, 2006). En estos términos, por ejemplo, las reservas hídricas -con las que trataremos más adelante-, son jurisdicciones territoriales. Sin embargo, como veremos, en el contexto de la política de los recursos hídricos se ponen en juego dinámicas espaciales que no resultan necesariamente en territorios delimitados, pero que forman parte indisoluble de la producción social de éstos. Por tal motivo elijo mantener el par conceptual espacio-territorio.

Córdoba es una de las 23 provincias que componen la República Argentina. Situada en la región centro, y con $165.321 \mathrm{~km}^{2}$ de extensión, es la quinta provincia más extensa del país. Su capital es la segunda ciudad con mayor cantidad de habitantes, luego de la Ciudad Autónoma de Buenos Aires, capital de la Argentina. La zona de las Sierras Chicas que abarca nuestra investigación se corresponde con parte de un corredor, tal como se suele referir a dicha zona en los medios de comunicación, cuyo componente urbano se extiende unos $45 \mathrm{~km}$ hacia el norte de la Ciudad de Córdoba y se corresponde con la traza de la Ruta Provincial E53, uno de los principales accesos a las sierras desde la Ciudad de Córdoba (Figura 1).

Figura 1 - Referencia espacial del área de estudio

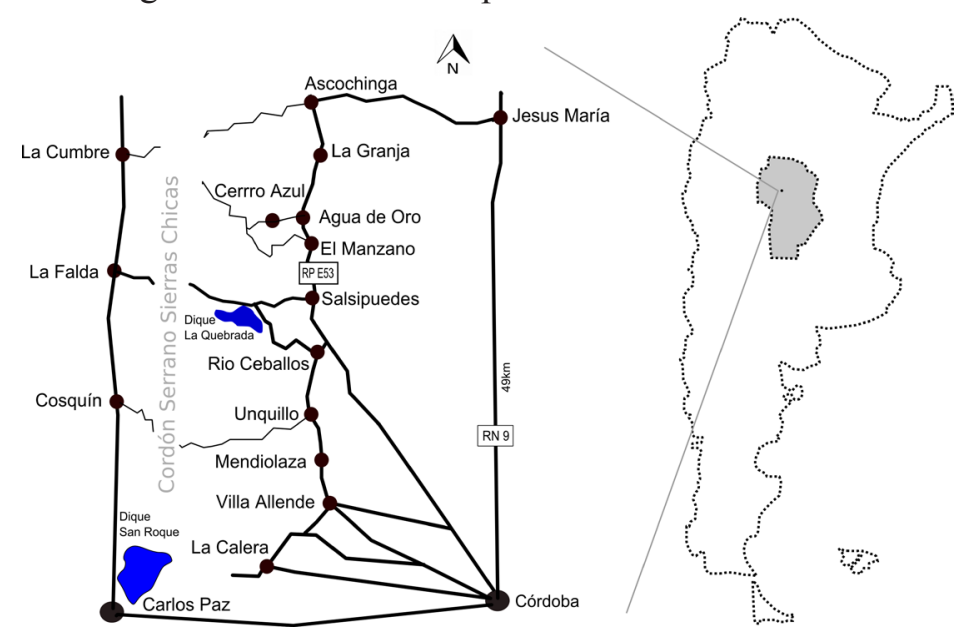

Fuente: elaboración propia del autor 
Como decíamos, nuestra investigación abarca una parte de dicho corredor dentro del cual se encuentran cinco Municipios y dos Comunas. Las Comunas son unidades político-administrativas autónomas que se diferencian de los Municipios en virtud de su cantidad de habitantes y la organización de su gobierno. Según datos del censo provincial del año 2008, La Comuna con menor cantidad de población permanente en la zona de Sierras Chicas abarca da por nuestro estudio cuenta con 220 habitantes, y el Municipio con la mayor cantidad cuenta con 19.133. En términos fisiográficos,

La Sierra Chica supera los mil metros de altura (cerro Pan de Azúcar 1257m); con pendientes dominantes entre 12 y $45 \%$ y un piedemonte de ondulaciones suaves. [...] Los suelos del Dominio Serrano se desarrollan en valles y pampas cubiertos por sedimentos gruesos, arenosos y loessoides, con afloramientos rocosos. [...] La ladera oriental presenta suelos de laderas escarpadas muy pedregosos y de laderas muy colinadas, pobres en materia orgánica y susceptibles de erosión hídrica y suelos de vallecitos de altura provistos de materia orgánica. (TAMBURINI y KUFNER, 2008, p.7)

En un sentido socio-espacial, las sierras chicas están plagadas de tensiones y contradicciones. $\mathrm{Su}$ ambiente natural es, histórica y actualmente, la base de una amplia diversidad de actividades productivas que generan dinámicas muchas veces antagónicas entre sí. En las sierras conviven la actividad pecuaria, la actividad de las canteras, la industria turística, la actividad inmobiliaria y la construcción, por nombrar algunas. Algunos pueblos y localidades de las sierras están transformándose paulatinamente en 'pueblos dormitorio'. Es decir, lugares que reciben a familias y habitantes que deciden vivir fuera de la ciudad capital, pero que mantienen vínculos permanentes con ella en virtud de las actividades laborales, educativas, etc. Las sierras son fuente de agua y otros recursos naturales que sostienen aquellas actividades productivas $\mathrm{y}$ dinámicas poblacionales que he mencionado $\mathrm{y}$, en virtud del sostenido crecimiento poblacional, el tema de los recursos hídricos disponibles es objeto de un amplio debate público en toda la zona, siendo motivo de preocupaciones de personas e instituciones que trabajan para enfrentar los problemas naturales y sociales que de se derivan de ello.

La investigación dentro de la cual se enmarca este artículo apela a diferentes metodologías y formas de indagación. Si bien centrada en la etnografía, recurro también a documentos escritos de diverso tipo, y al análisis comparativo -tal como la antropología entiende estas diferentes herramientas metodológicas-. La etnografía, como método cualitativo de investigación social, se basa en un trabajo de campo centrado en la observación participante y la entrevista abierta, técnicas que implican el contacto directo del investigador con los protagonistas de las relaciones y procesos sociales que pretende analizar. Tanto la diversidad de las formas que adoptan estas relaciones y procesos, como las perspectivas que sobre los mismos tienen sus protagonistas, constituyen la base de todo análisis propiamente etnográfico. (BALBI y ROSATO, 2003). Una característica fundamental del tipo de análisis que adoptamos es la de incluir la comparación entre sus herramientas. La comparación, utilizada en el curso mismo del análisis etnográfico, permite hacer de la etnografía una instancia analítica, no meramente descriptiva, dando así pleno valor a la indagación intensiva desarrollada en el campo (PEIRANO, 1995; BALBI y ROSATO, 2003). En este sentido, recurro a dicha herramienta a fin de establecer las dimensiones de variabilidad (BARTH, 2000) a que se encuentran sujetos los procesos a analizar.

La argumentación conceptual y teórica de este artículo gira en torno a la manera de tratar al espacio y al territorio en relación a la política de los recursos hídricos. En función de ello, exploraré a continuación ciertas perspectivas espaciales $\mathrm{y}$ territoriales que fueron elaboradas desde la antropología en particular; dicho recorrido me permitirá asimismo sentar los fundamentos de la elección del enfoque teórico tomado para el análisis. 


\section{El espacio-territorio en antropología. Algunos antecedentes}

La antropología ha tenido, desde sus inicios profesionales, una relación ambigua y contradictoria con el espacio en tanto objeto de análisis. Nacida de una representación ideológica de la estructura centro / periferia, y marcada por una posición de la mirada que se colocaba 'en' el centro y 'desde' el centro, la relación con la periferia fue entendida en términos de una secuencia evolutiva que colocaba a Europa, el centro, en la cúspide de la evolución socio-cultural. De esta manera, la antropología del siglo XIX pecó de una errónea traducción del espacio al tiempo (FRIEDMAN, 1994). Para la misma época, el paradigma difusionista colocó a las temáticas espacio-territoriales en relación con la determinación de áreas culturales, regiones sociopolíticas o regiones económicas (THER RIOS, 2006). Luego de la superación de dichos paradigmas, la cuestión espacial fue perdiendo peso en la antropología. En obras posteriores al difusionismo, el espacio aparece relacionado al medio ambiente como un elemento de la descripción etnográfica o como información contextual, perdiendo su importancia en relación con la producción y reproducción de la vida social (RANKLIN, 2003). Hubo que esperar hasta la publicación de Los Nuer de Evans-Pritchard en 1940 -una obra que se esfuerza por integrar el análisis de la estructura social con el de la ecología (THER RIOS, 2006)- para que se volviera a problematizar en profundidad la cuestión espacial, una preocupación que también se refleja en la obra de Pitt-Rivers (1994), quien fuera discípulo de Pritchard.

La temática espacial, aunque con otros matices, ha tomado un nuevo impulso a partir del interés suscitado por los procesos de conformación de 'bloques regionales' de fines del siglo XX. La Unión Europea, por ejemplo, se ha convertido en objeto de análisis antropológico (ABÉLĖS, 2004; CHAPMAN, 1994; SHORE, 2000, entre otros). En Latinoamérica, la renovada atención a los análisis de corte territorial en antropología se debe, en gran parte, a dos tipos de procesos: en primer lugar, a los procesos de integración regional encarados por los Estados Nacionales y Provinciales hacia fines del siglo XX. En segundo lugar, a la creciente visibilización de los conflictos étnicos por el control y apropiación del territorio.

En cuanto al primer fenómeno, cabe destacar como ejemplo la firma el "Protocolo 23" en 1988, denominado "Protocolo de Integración Regional Fronteriza" que fuera incorporado luego al MERCOSUR. Este protocolo marca el comienzo de un proceso de integración regional entre la provincia argentina de Entre Ríos y el estado brasileño de Rio Grande, cuya institucionalización fue analizada por Rosato (2009). Por su parte, Balbi, Rosato y Boivin (2009) analizan dicho proceso en términos de la integración como representación social. Otro ejemplo de este tipo de dinámicas es el lanzamiento oficial, en el año 2004, de la Región Centro de la República Argentina que fue analizado por Gaztañaga (2008), quien afirma que el proceso "representa un entramado de problemas operativos y normativos cargados de reclamos históricos, imaginaciones colectivas y axiologías acerca del proceso regional" (GAZTAÑAGA, 2008, p. 237). Así, la región es entendida como involucrando espacio-territorios que "siendo físicos son también de producción y reproducción de relaciones sociales e imaginaciones colectivas" (HEREDIA, 1999, p. 85).

La segunda línea es mucho más prolífica que la primera y es imposible hacer justicia a la gran cantidad de trabajos antropológicos publicados que, desde hace décadas, analizan los conflictos entre las poblaciones originarias y los Estados Nacionales en torno al reconocimiento de los derechos de ocupación y apropiación del territorio. Por cuestiones de extensión haremos mención sólo a un par de la incontable serie de publicaciones. Así, por ejemplo, Pacheco de Oliveira (2010) afirma que en cuanto a las políticas estatales se refiere, "la categoría 'tierra indígena' no se corresponde, per se, a una descripción sociológica, sino mas bien a una categoría jurídica". Analizando este tipo de conflictos en la zona del Amazonas, Little (1997) propone los conceptos de "territorio social" y de "cosmografías" para entender la forma en que el territorio es producido y reproducido conflictivamente a través de la apropiación desigual de la geografía. Por su parte, Briones (2005) aborda la configuración del espacio 
social en diversas provincias de la Argentina en relación a la problemática indígena en ese país.

En todos los análisis sobre territorialidad indígena $\mathrm{u}$ otras temáticas que giran alrededor del espacio y el territorio (cabría mencionar también a la antropología urbana), éste es considerado en términos del sustrato espacial necesario de toda relación social, pero implicando cuestiones mucho más amplias que las condiciones y determinaciones físicas o naturales del espacio.

De nuestro análisis en torno a las dinámicas de los recursos hídricos en las sierras de Córdoba también se desprende que el espacio-territorio es producido y reproducido en el marco de procesos conflictivos que no pueden explicarse exclusivamente en términos fisiográficos, pues éste adopta diferentes formas en función de la dinámica de aquellos conflictos. En esta línea, hay una cuestión sobre la cual quiero reflexionar aquí, y que refiere a la relación entre los procesos espacio-territoriales y cierto producto que resulta de ellos: las formas fijas y fijadas con intenciones permanentes. Siguiendo a Harvey (2009), considero que, para entender al espacio-territorio como un proceso social en constante cambio y transformación, deberíamos explicar asimismo su fijeza y su permanencia en el tiempo. Esta idea no es sino la aplicación, a la problemática del espacio, de los desarrollos de Marx (1994 [1867]) acerca de la circulación y reproducción del capital.

La comprensión de la relación entre ambas dimensiones (permanencia / transformación) puede aportar a un mayor entendimiento de las diferentes lógicas, dinámicas y procesos espacio-territoriales específicos. Creemos necesario, además, sumarle a esta perspectiva una cuestión que se nos reveló durante nuestro trabajo de campo etnográfico. Nos referimos, concretamente, al carácter intencional con que diversos actores pretenden incidir sobre las formas espacio-territoriales en el marco de una serie de conflictos socio-ambientales que giran alrededor de los recursos hídricos.

El eje de nuestra exposición son las formas en que el espacio-territorio se manifiesta y se expresa en el marco de la política de los recursos hídricos en sierras chicas. En primer lugar, entonces, presento estas formas, para luego desarrollarlas y describir cómo influyen en los procesos a analizar. Estas son: a) el espacio-territorio concebido como algo 'dado', es decir como objetivo; b) como algo definido en función a la posición del observador, es decir, como diverso; y c) como relación y proceso social. Consideramos que la tensión entre estas diferentes formas en que se manifiesta el espacio-territorio es un elemento central para comenzar a explicar su especificidad en relación a los recursos hídricos. Cabe aclarar que son formas que no son separables más que analíticamente. No se encuentran aisladas ni inconexas, aunque podría parecerlo, debido a que así se nos presentan a la experiencia. Cada una de estas formas se corresponde con el espacio considerado como absoluto, como relativo y como relacional que propone David Harvey (2006).

Dijimos que comenzaríamos a buscar la especificidad de la relación entre el agua y el espacioterritorio a partir de explorar la manera en que las tres formas mencionadas se ponen en tensión en el marco de la política de los recursos hídricos en sierras chicas. Como mostraremos más adelante, en algunas ocasiones se trata de una puesta en tensión explícitamente intencional y planificada por parte de los actores; otras veces es menos explícitamente planificada, aunque no por ello menos intencional; en otras ocasiones, la tensión se deriva de las acciones de los actores, aunque sin necesariamente tener una intencionalidad en ese sentido. En función de estas consideraciones, estamos en condiciones de afirmar que tratamos con procesos de producción social del espacio-territorio. Afirmamos, asimismo, que la política de los recursos hídricos implica y conlleva necesariamente dicha producción.

Ahora bien, como toda producción humana es social, podemos obviar la redundancia y hablar de producción, a secas, del espacio-territorio. En función de lo dicho planteamos, en primer lugar, el contexto que nos lleva a preguntarnos acerca de la relación entre los recursos hídricos y el espacio-territorio. En segundo lugar, prestamos atención a la manera en que los actores conciben esta relación, para finalmente proponer una forma de encarar analíticamente las formas diferenciales que ella asume. 


\section{La crisis hídrica en Sierras Chicas}

El problema del agua ha estado presente en la agenda pública de la provincia de Córdoba desde hace más de una década. En las sierras chicas en particular, comenzó a manifestarse como uno de los efectos de la falta de lluvias, del sostenido crecimiento poblacional de las localidades de la zona, y de la falta de inversión en infraestructura por parte del Estado, entre otras razones. A partir de entonces, la provisión del recurso para las poblaciones locales se volvió objeto de una serie de debates que giran alrededor de las causas, las responsabilidades y las soluciones a una situación que se vivió y se vive como una crisis. En algunos lugares y durante ciertos períodos, dicha situación ha llegado a ser considerada en términos de una emergencia.

En los medios de comunicación de Córdoba, las noticias respecto de la falta de agua en las Sierras Chicas son moneda corriente. Fotos de arroyos secos, de embalses muy por debajo de su nivel normal, avisos de multas a derrochadores y cronogramas de cortes del servicio en distintas localidades, ofrecen el contenido a títulos tales como:

"Cada vez hay más pueblos con mucha sed" (Día a Día, 29/12/10).

"Habrá multas [...] por lavar veredas o autos en Río Ceballos" (La Voz, 01/10/11)

"Sin lluvias y con los diques 10 metros abajo" (Día a Día, 03/10/11)

"El secazo. Salsipuedes entró en alerta roja" (Día a Día, 4/10/11)

"Dos mil familias reciben agua sólo una vez por semana" (La Voz, 18/10/11)

"Reunión clave en Salsipuedes para enfrentar la grave crisis hídrica" (Día a Día 20/01/14)

"A pesar de las lluvias, no hubo agua en las Sierras Chicas" (Día a Día, 17/02/14)

Las iniciativas para enfrentar la crisis involucran tanto a funcionarios y políticos de los distintos niveles de la jerarquía burocráticoadministrativa del Estado, como a cooperativas de servicios públicos, organizaciones no gubernamentales (en adelante ONG), vecinos agrupados, y también usuarios que reclaman agua potable cuando hay faltante. En los últimos dos años, además, se han creado nuevas y se han fortalecido viejas agrupaciones que inciden en la política de los recursos hídricos, y que propician revertir todo aquello que incida negativamente sobre la calidad y la existencia del recurso, así como en el medio ambiente en términos generales. Entre otras actividades, impulsan campañas de concientización ambiental a través de talleres en las escuelas, organizan festivales por el agua y el ambiente, presentan proyectos ante las autoridades municipales y provinciales, e incluso han llegado a manifestarse a través de cortes de ruta y otras medidas políticas para llamar la atención de los gobiernos locales, las agencias del estado provincial y de la población en general.

En lo que refiere al agua potable en varias localidades, el servicio de red domiciliaria está concesionado a cooperativas de servicios públicos. Algunas fueron creadas en el marco del Plan Nacional de Agua Potable hacia mediados de la década de 1980. Otras cooperativas surgieron por iniciativa de los vecinos frente a la ausencia del Estado en cuanto a lo referido a los servicios públicos. Algunas cooperativas se encargan de la red de un paraje o villa, o de un sector de una localidad. Otras abarcan a más de una localidad. En ciertos lugares, es el Municipio mismo quien se encarga de la distribución del agua potable a los consumidores finales. Según la historia de cada una de ellas, las cooperativas pueden tener concesión del Municipio o de la Provincia. No nos detendremos en analizar estos detalles jurídicos aquí; simplemente los mencionamos con la intención de destacar la gran variabilidad que existe en la zona en cuanto a la organización del servicio de agua.

A esta variabilidad se le suma aquella relativa a los procesos técnicos de captación y potabilización del agua. Dos de las localidades más importantes de la zona se proveían, hasta hace poco, del Dique La Quebrada que cae bajo jurisdicción del gobierno provincial. Este dique se encuentra en una reserva hídrica que lleva por nombre Reserva Hídrica Natural Parque La Quebrada. Actualmente, estas localidades comenzaron a proveerse en forma paulatina del Dique San Roque ubicado en la localidad de Carlos Paz, que también provee de agua potable a parte 
de la ciudad de Córdoba, la segunda ciudad más poblada de la Argentina. Aunque el agua provista por medio de embalses reviste una importancia mayor en términos de cantidad y volumen, no se trata de la única fuente. En ciertas localidades de las sierras el agua se obtiene a través de captaciones en los arroyos o de los reservorios, como es el caso de Agua de Oro; en otras a través de pozos, como es el caso de Salsipuedes. En los últimos años, este Municipio fue reiteradamente declarado bajo emergencia hídrica, ya que los aproximadamente doce pozos desde los cuales se capta el agua de napas subterráneas están secándose paulatinamente. En el año 2009, Salsipuedes se transformó en un caso paradigmático de la crisis hídrica desde el momento en que circuló una noticia que afirmaba que las cisternas con las cuales se repartía el agua en camiones en momentos de faltante, debieron ser custodiadas por la policía debido a posibles enfrentamientos entre los vecinos por una porción de agua (Diarios La Voz y Clarín, 10 de noviembre de 2009).

Como puede apreciarse, la organización del la captación, producción y distribución del agua potable en la zona es muy variable, así como las intensidades en que cada localidad vive la crisis. Esta variabilidad es relativa, entre otras cuestiones, a la participación de las agencias estatales provinciales y locales, a los procesos técnicos que implica la provisión de agua desde diferentes fuentes (embalses, pozos, reservorios) y a los antecedentes históricos de cada lugar. Lo que le da unidad a esta diversidad es el hecho de que toda la zona de sierras chicas se enfrenta a una crisis hidrica que es preocupación tanto de los consumidores como de los responsables de la producción y distribución del agua potable, así como de políticos y funcionarios, ONG ambientalistas y agrupaciones de vecinos.

\section{La política de los recursos hídricos: crisis, conflictos $y$ territorios}

Los actores involucrados en la política de los recursos hídricos aportan iniciativas para enfrentar la crisis desde diversos planos articulados entre sí. Uno de ellos refiere al control sobre el consumo de agua; otro a las iniciativas de ordenamiento territorial, y otro a las propuestas regionales de acción. Por cuestiones argumentales y de espacio, aquí dejaremos el tema del consumo de lado.

El ordenamiento territorial refiere a la reglamentación del uso del suelo en función del impacto que dicho uso tenga sobre el recurso hídrico en particular y sobre el medio ambiente en general. Es considerado por muchos como uno de los pilares para enfrentar la crisis. Al respecto sobresalen, por ejemplo, iniciativas como la creación de reservas hídricas y el manejo de las ya existentes, el control de la construcción indiscriminada y del avance inmobiliario, del sobre-pastoreo y la deforestación, el control de los incendios forestales, el cuidado del consumo de agua potable, el financiamiento de obras de infraestructura, la regulación de la actividad turística, etc. Como iniciativa del Estado así como de ONG y agrupamientos varios, el ordenamiento territorial es un ámbito particularmente conflictivo debido a que implica decisiones proyectadas hacia un futuro, pero que influyen inmediatamente en la vida cotidiana de las personas así como en el ámbito jurídico, político y económico. Asimismo, el reconocimiento de los aspectos comunes de las diferentes localidades de la zona en lo que refiere a la crisis hídrica, impulsa a muchos a encarar iniciativas regionales que exceden las unidades político-administrativas locales en función de buscar y encontrar soluciones abarcativas que se tomen en conjunto.

Uno de los problemas centrales del ordenamiento territorial son las reservas hídricas. Según nuestros datos, la más antigua en la zona es la Reserva Hídrica Natural Parque La Quebrada, de unas 4200 hectáreas, creada en 1987 sobre tierras privadas y ubicada en las inmediaciones de la localidad de Río Ceballos. La reserva tiene tres patas, nos comentaba un funcionario de la Municipalidad: la Dirección Provincial de Vialidad, la Subsecretaría de Recursos Hídricos (también un organismo provincial) y la Municipalidad a través de su Dirección de Ambiente, que forman un Concejo Asesor que debería reunirse, por convenio, cuatro veces por año. Dentro de este parque - reserva se encuentra el dique La Quebrada, que tiene varios usos: turísticos, control de crecidas, y captación de agua para su posterior potabilización. 
La superficie del espejo de agua es de 35 ha a nivel del vertedero. El volumen del embalse a cota del labio vertedero es de $3.3 \mathrm{hm}^{3}$, siendo uno de los diques más 'pequeños' de la provincia, comparado con el resto. Para visualizar mejor la diferencia, mencionemos a uno de los diques más conocidos de Córdoba, el dique San Roque, ubicado en la ciudad de Carlos Paz, que tiene una capacidad para embalsar unos $201 \mathrm{hm}^{3}$ de agua. Este dique provee de agua a parte de de la Ciudad de Córdoba, y actualmente a algunas de las localidades de las Sierras Chicas.

Si bien la jurisdicción efectiva sobre el territorio de la reserva La Quebrada recae sobre el estado provincial, la Dirección de Ambiente de la Municipalidad de Río Ceballos había logrado que le delegaran la potestad de controlar el perilago. La incidencia de la Municipalidad sobre la reserva es limitada, pues si tenemos en cuenta que la superficie total es de 4200 hectáreas, el perilago es sólo una 'pequeña' porción. La Provincia se encarga del resto a través de un destacamento de guardaparques. En el territorio de la reserva existe un estricto control sobre la propiedad, específicamente en lo que refiere a la subdivisión para su venta y a la superficie de edificación permitida. Es necesario regular la propiedad privada, pues la reserva es fuente de agua para miles de personas, nos comentaba un especialista en el tema. De esta manera, el bien común debe primar sobre el interés y la propiedad. Esta relación entre interés, propiedad privada y bien común, es uno de los puntos del debate sobre la crisis hídrica. En función de esto, el actor más interpelado como el responsable de una solución es el Estado Provincial.

Además de la reserva La Quebrada, en las sierras chicas existen otras reservas hídricas. Algunas de reciente creación, otras se remontan atrás en el tiempo. Varias de ellas se encuentran a medio camino. Es decir, están creadas legalmente, pero no reglamentadas. Y este punto es, justamente, objeto de demandas hacia el Estado en varias localidades, pues se trata de un tema que coloca la situación jurídica de la tierra en primer plano. Como se puede apreciar, las reservas aparecen como delimitaciones jurisdiccionales con reglas propias. Al establecer límites, establecen entonces una diferencia, y pueden entenderse como una forma específica de control y apropiación del territorio. En este caso tratamos con reservas hídricas, y el control sobre el territorio viene acompañado del control sobre el recurso. Veamos otros ejemplos.

Las reservas pueden ser también Municipales o Comunales. Varias reservas fueron creadas como resultado del trabajo y las iniciativas de ONG. Sin embargo, en muchas ocasiones el paso siguiente en términos legales -la aprobación y reglamentación de los planes de manejo- demora en realizarse, un aspecto que es considerado como un problema grave en cuanto a la puesta en práctica efectiva de las reservas. Un plan de manejo es, sintéticamente, el conjunto de normativas que regulan lo que se puede hacer o no dentro de una reserva.

Cuando las iniciativas para la creación de una reserva provienen de las autoridades, tal como sucedió en una de las Comunas de la zona, la reglamentación del plan de manejo no es un problema. A veces sucede que el territorio de la reserva coincide plenamente con un ejido comunal, situación en la cual pueden enfrentarse lógicas territoriales contradictorias. En uno de los casos en que se da esta situación, sucedió que la ampliación de una calle generó tensiones hacia el interior del gobierno comunal debido a que los escombros resultantes habrían caído hacia un arroyo. Según interpretaron algunos miembros del gobierno, se trató de una violación del plan de manejo de la reserva.

$\mathrm{Si}$ entendemos las reservas como jurisdicciones que producen formas diferenciales de apropiación y control, toma sentido el conflicto. En otras circunstancias, ampliar una calle es un acto de gobierno legítimo para cualquier unidad políticoadministrativa autónoma como las Comunas, y proteger el arroyo es una acción legítima en el marco de una reserva. Pero en esta situación, la ampliación de una calle entró en conflicto con una lógica territorial diferente, lo que produjo que sea considerada como 'ilegítima' por parte de un sector del gobierno comunal. Podemos afirmar entonces que, en este ejemplo, existen dos lógicas territoriales que se extienden geográficamente sobre la misma superficie.

Una situación homóloga, aunque en una escala mayor, sucedió con el retrazado y 
pavimentación de la Ruta Provincial E-57 que cruza las sierras desde la localidad de Salsipuedes hacia el valle conocido como el Valle de Punilla, y cuyas obras de pavimentación y retrazado se sucedieron entre los años 2005 y 2011. Dos reservas habrían sido afectadas por los impactos ambientales de esta obra. Del lado oriental de la sierra, la reserva La Quebrada ya mencionada. Del lado occidental, la reserva Vaquerías, que abarca unas 400 hectáreas de tierras pertenecientes a la Universidad Nacional de Córdoba. Según una noticia de prensa, el estado provincial tuvo que invertir 20 millones de pesos -a razón de 4,28 pesos por dólar estadounidense, en aquella época- para remediar el impacto ambiental producido sobre esta reserva, siendo la primera vez que es reconocido un daño ecológico a partir de una obra de esa envergadura. Sucedió así, afirma la nota, debido a que buena parte de los reclamos fueron planteados a la Provincia por la Universidad Nacional de Córdoba (Diario La Voz 18/11/11, versión electrónica).

La intervención, en el año 2006, de un organismo del poder ejecutivo provincial, -la Gerencia de Áreas Naturales Protegidas, dependiente de la Agencia Córdoba Ambiente-, fue también significativa en este conflicto. Elevó un informe al gobierno provincial en el cual se recomendaba dar participación a la Universidad Nacional de Córdoba en la evaluación de los impactos ambientales. Nos encontramos, nuevamente, ante un caso que nos presenta lógicas territoriales diferentes, pero que se extienden espacialmente sobre un mismo área. El conflicto es, por lo tanto, inevitable.

\section{El territorio como una jerarquía de niveles}

Esta primera aproximación a la diversidad de actores y complejidad de dinámicas alrededor del recurso hídrico nos permite comenzar a explorar la especificidad de la relación agua / territorio. Centrémonos en primer lugar en los actores. Hasta aquí, y en lo que refiere a los conflictos que giran en torno las reservas hídricas, podemos resaltar el protagonismo de las agencias del Estado. Al respecto también se destaca que tratamos con agencias que operan en diferentes niveles de la jerarquía burocrática. Además, los conflictos que hemos descrito implican diferentes niveles territoriales, al menos en términos de jurisdicción e influencia: desde una Comuna hasta el Ejecutivo Provincial; desde la Comisión de Ambiente de una Comuna, pasando por la dirección de Ambiente de un Municipio y llegando hasta la Gerencia de Áreas Naturales Protegidas de la Provincia.

En un principio, habíamos entendido que la dinámica e interacción jerárquica entre estos niveles territoriales considerados como jurisdicciones, habría de ser determinante en el desarrollo de la política de los recursos hídricos (KOBERWEIN, 2013). Sin embargo, hoy creemos que esto es cierto sólo en parte. En primer lugar, porque no hemos encontrado evidencias de que, en el marco de la política de los recursos hídricos, el territorio se organice exclusivamente en términos de niveles jerárquicos, o en cualesquiera otros términos que nos permitan afirmar tal cosa. En segundo lugar, si bien la jerarquía territorial - jurisdiccional históricamente establecida puede entrar en contradicción con otras formas en que se manifiesta o se produce socialmente el territorio, el hecho de que éste se organice en forma jerárquica es resultado de procesos no necesariamente relacionados con los recursos hídricos. Por lo tanto, la especificidad de la relación agua / territorio debe buscarse por otro lado.

Como hemos hecho notar, las iniciativas referidas al manejo de la crisis hídrica están enfocadas en la intervención planificada sobre la organización espacio-territorial. Esta intervención implica, entre otras cuestiones, la creación de reservas hídricas y la regularización del manejo y el uso de las ya existentes. Como decíamos, al establecer límites, las reservas hídricas crean una diferencia; en principio, en términos de la apropiación del territorio. Pero sobre todo, y al tratarse de reservas hídricas, una diferencia en términos de la manera en que el recurso hídrico es apropiado. Las contradicciones y conflictos en torno a las competencias de las diferentes agencias del Estado que hemos mencionado más arriba pueden deberse al estatus jurídico 'especial' de las reservas. Una hipótesis que puede plantearse es que los conflictos y discontinuidades que hemos mencionado pueden deberse en parte al hecho de que la tierra en las reservas puede comercializarse, aunque no libremente, siendo que el mercado requiere, idealmente, de 'libertad' plena para comprar y vender. Sin embargo, aquellas contradicciones y 
conflictos no son exclusivas de las reservas hídricas. Para dar cuenta de ello deberíamos considerar otros territorios.

La unidad territorial / jurisdiccional más amplia dentro de la Provincia de Córdoba es el departamento. Los departamentos tienen representación en la legislatura de la Provincia. Cada departamento está subdividido en pedanías, que son unidades que tienen una función catastral. Las unidades territoriales subsiguientes, en este orden de escala descendente, son los Municipios y luego las Comunas. Finalmente, el paraje, el caserío o la villa, son también consideradas como unidades espaciales, aunque dependientes ya sea de algún Municipio, de alguna Comuna o incluso de la Provincia, aunque esto fue siempre fuente de disputas entre unidades políticas locales.

En las últimas décadas ocurrieron una serie de conflictos limítrofes entre Municipios y Comunas en torno a la definición de sus ejidos. En este marco es posible que, con el tiempo y a través de iniciativas políticas diversas, un caserío o villa pueda transformarse en Comuna. En la Provincia de Córdoba hay sobrados ejemplos de ello, tanto frustrados como exitosos. Las zonas serranas fueron especialmente sensibles a estos conflictos debido a que se han ido poblando cada vez más. Zonas que antes no eran reclamadas por ninguna unidad política local como perteneciente a su jurisdicción comenzaron a serlo y, cuando dos Municipios, por ejemplo, pretendían la misma zona, no hace falta aclarar que el conflicto comenzaba a fluir como el agua misma.

Un conflicto representativo de este tipo, actualmente resuelto, tuvo como eje la relación recursos hídricos / territorio. Discurrió durante varios años entre un Municipio y un sector de vecinos de un paraje ubicado $5 \mathrm{~km}$ sierra arriba del casco urbano de la localidad. Un sector de vecinos proponía que el paraje se independizara del Municipio para formar una Comuna autónoma. Para conseguir la autonomía, los comuneros -tal como los llamaban algunosactuaron políticamente desde la cooperativa de agua en dicho paraje. Una de las tantas y variadas versiones que circularon y que explicaron la oposición del Municipio a dicha autonomía fue la posibilidad de que una reserva hídrica quedara ubicada en territorio comunal. El poder ejecutivo provincial, luego de varios años de conflicto entre el gobierno municipal y los vecinos del paraje, finalmente no autorizó la creación de la Comuna. De esta manera, el territorio se nos presenta como algo dinámico y conflictivo, tal como ya han demostrado por ejemplo Simmel (1939), Lefebvre (1974), Santos (1990) o Harvey (1997, 2006 y 2009), por nombrar sólo a algunos de los más influyentes al respecto.

He mencionado que las iniciativas para enfrentar la crisis hídrica pretenden incidir sobre el territorio. En principio, ordenándolo. También he mostrado que en esta dinámica, las jurisdicciones y competencias de las agencias del estado sobre el territorio entran en contradicción. Estas contradicciones se ven alimentadas no solo en función de una superposición de las competencias de las agencias estatales. Además, se cruzan con la organización jerárquica del Estado. Hay variantes en torno a la interacción entre jerarquía y competencia. Puede ser que las acciones de dos agencias del Estado que tienen la misma jerarquía impliquen un conflicto de competencias dentro de un mismo territorio. Podríamos pensar los conflictos entre la Dirección Provincial de Vialidad y la Agencia Córdoba Ambiente en torno a los impactos del trazado de la ruta E57, como una variante de este tipo. Puede ocurrir que dos agencias de distinta jerarquía entren en conflicto por competencias sobre un mismo territorio; o puede también darse el caso en que dos unidades políticas locales del mismo estatus (dos Municipios, por ejemplo) entren en conflicto por una misma porción de territorio, o que un caserío o villa le dispute competencias a un Municipio al querer independizarse de éste.

Lejos estamos de pretender realizar una clasificación de estas variantes. Las hemos mencionado porque ilustran que el espacio-territorio es dinámico y fuente conflictos. La tensión entre diferentes agencias estatales en función de sus competencias y jerarquías, así como los conflictos entre las unidades políticas locales, son sólo dos formas que asume la dinámica espacio-territorial. Pero esta explicación no pasa de ser una explicación técnica que resulta de pensar en términos técnicos, por no decir tecnocráticos. Es decir, en términos de la organización y del funcionamiento del Estado y el gobierno, de sus incidencias y efectos sobre el territorio o los recursos hídricos. El lenguaje de la técnica es el de la causa y el efecto, el de las responsabilidades y de las previsiones, el de la ley y 
la propiedad, el de las competencias y las jerarquías. Es, digámoslo en los términos más generales posibles, el mundo de lo fijado con intenciones permanentes: un camino, un embalse, una red de distribución de agua potable, una reserva hídrica, los límites de un Municipio, etc. Un mundo con reglas claras, aunque, tal como hemos visto, marcado por el conflicto.

Se trata de lo que Harvey (2006) denomina el espacio absoluto, aquél espacio en el cual se pueden planear y regular eventos, acciones $\mathrm{y}$ comportamientos. El espacio absoluto por excelencia es el de la propiedad privada. Pero también incluye otras designaciones territoriales con límites, tales como los estados y las unidades administrativas. En este espacio absoluto, "todas las incertidumbres y ambigüedades pueden ser anuladas, y el cálculo puede florecer sin restricciones"[original en inglés, traducción propia] (HARVEY, 2006, p. 272). Se trata, en síntesis, del mundo de lo planificado y de la posibilidad de planificación. Pero la forma absoluta no es la única manera en que se manifiesta el espacio. De las mismas situaciones presentadas anteriormente, específicamente de aquellas que involucran conflictos concretos, podemos también hacer referencia a otras formas. Desde esta perspectiva, y concebido como proceso, el espacio se nos presenta como territorio únicamente en sus formas absolutas. Sin embargo, las formas absolutas están estrechamente vinculadas con otras formas espaciales y no pueden entenderse en su especificidad sin hacer referencia a ellas. En este sentido, no todo espacio es entendido como un territorio, pero todo territorio (es decir, todo espacio absoluto) es aprehensible analíticamente -desde la perspectiva aquí adoptada- sólo como producto de procesos espaciales no absolutos. De allí que el territorio pueda ser entendido como un momento, o una fase de la producción social del espacio en general.

\section{La relatividad del espacio-territorio}

Si nos salimos de la perspectiva tecnocrática, nos enfrentamos con el mundo del espacio diverso y relativo. Hasta el momento estábamos tratando con la forma absoluta del espacio: reservas hídricas, Comunas, Municipios y jurisdicciones que tienen límites establecidos socialmente. La forma absoluta se nos manifiesta en primer plano sólo si pensamos en términos técnicos. Si damos un paso más allá, lo absoluto se relativiza y se transforma en diverso, todo lo contrario a algo absoluto.

En este sentido, el mismo territorio puede ser una reserva hídrica o un ejido comunal, dependiendo de la posición de la mirada. Pero podemos ir más lejos aún y decir que para el turista en auto o el caminante, para el productor ganadero, para el dueño de una cantera o para quien se siente a contemplar el paisaje al atardecer, el espacio-territorio se la va a manifestar de maneras muy distintas. "Implica múltiples geometrías entre las cuales elegir y el marco espacial depende crucialmente de qué es lo que se está relativizando y quién lo hace". [original en inglés, traducción propia] (HARVEY, 2006, p. 272). De absoluto, el espacio se transforma así en relativo y diverso.

Para el caso del trazado de la ruta E57, la geometría que 've' la Gerencia de Áreas Protegidas -que tiene por función evitar el impacto ambiental negativo en una reserva-, no es la misma geometría que 've' la Dirección de Vialidad Provincial, que debe dinamitar la sierra para trazar una ruta. Cuando una 'mirada' entra en conflicto con otra, estamos en el ámbito de lo político.

A priori, y tal como hemos tratando al territorio en la sección anterior, este se organizaría en una suerte de jerarquía inclusiva. Para David Harvey (2007) estos ordenamientos jerárquicos del espacio dan forma a una escala. Sin embargo, afirma el autor, esta definición de escala tiene un riesgo: es muy parecida a su definición intuitiva, mucho más cercana a las concepciones absolutas del espacio que a concepciones relativas y relacionales. Sin embargo, el problema no es la jerarquía. El problema es tomarla como dada, natural e inmutable. La idea de un territorio ordenado jerárquicamente tiene potencial analítico únicamente si consideramos este ordenamiento como el resultado de procesos sociales de producción espacio-territorial. Dicho 'ordenamiento', ¿es producto de qué procesos, de qué relaciones?, ¿cómo se produce?, ¿cuáles son las maneras en que esa jerarquía se mantiene en el tiempo o se pretende mantener?, ¿quién pretende mantenerla, quién modificarla?

Sólo si consideramos al territorio como no-absoluto podremos comprender la manera en que la jerarquía escalar 'absoluta' se produce. "La definición de dónde podría empezar y terminar un 'ecosistema' y qué tipo de 'entidad' podría estar en 
qué tipo de escala (¿una laguna o un continente?) es fundamental para la cuestión de cómo formular una política ecológicamente sensata" (HARVEY, 2007, p.96) Por lo tanto, estas escalas nada tienen de 'naturales'. En este sentido las escalas son, dice Harvey, productos de tecnologías, modos de organización y luchas políticas.

\section{El espacio-territorio como relación y proceso}

Para comprender más específicamente esta socio-política incorporemos el tiempo en el espacio. En la introducción habíamos comentado que estos procesos espaciales ligados al recurso hídrico ocurren en el marco de una triple tensión entre el espacioterritorio concebido como algo fijo, como algo diverso (o relativo) y como un conjunto de relaciones y procesos. Ya hemos mencionado las primeras dos formas. Nos queda la tercera, aquella que considera que el espacio-territorio no existe por fuera de los procesos y relaciones que lo definen.

Aquí no se trata de cómo 'ven' el espacioterritorio los actores y, por lo tanto, cómo actúan sobre él en función de esa 'mirada'. Éste se define por las relaciones entre ellos a lo largo del tiempo. $\mathrm{O}$, en otras palabras, se define por las relaciones y procesos que ocurren 'en' un espacio producido por esas mismas relaciones y procesos. En otras palabras, la perspectiva aquí adoptada nos impone que los procesos no ocurren en el espacio, sino que definen su propio marco espacial, como afirma Harvey. Veamos algunos ejemplos para ilustrar en qué medida sucede esto en Sierras Chicas y en relación a la política de los recursos hídricos.

En la localidad de Agua de Oro, uno de los conflictos actuales más importantes refiere a la autorización, por parte de las autoridades provinciales, de un loteo en las partes altas de la sierra que afectaría considerablemente la provisión y calidad del agua potable debido al aumento en el consumo que sobrevendría a la construcción de viviendas y la posible contaminación de la cuenca con aguas servidas. Junto con la cooperativa que se encarga de la captación y distribución del agua se han movilizado vecinos -en rigor los vecinos son socios de la cooperativa- que se han manifestado a través de cortes de ruta en contra de esos loteos, reclamando asimismo al Estado Provincial la participación de la cooperativa en los mecanismos burocráticos que los autorizan. Para difundir la problemática se filmó además un documental sobre el problema. Un conflicto similar ocurrió en el Municipio vecino de Salsipuedes. Las autoridades provinciales habían aprobado la factibilidad para la construcción de edificios de departamentos, aduciendo que los informes técnicos revelaban que, bajo los terrenos afectados a la construcción, existe agua suficiente para autorizar las obras. Actualmente este emprendimiento fue puesto en suspenso -debido, entre otras razones, a la intervención de una agrupación de vecinos de la localidad- $y$, en vez de un edificio de departamentos, fue construido un centro comercial-recreativo proyectándose también un futuro complejo hotelero.

Muchos actores consideran que el problema hídrico excede los límites de las unidades políticas locales. Esto hace que se propongan iniciativas de carácter regional para enfrentarla. Así, por ejemplo, varias autoridades municipales de la zona se reunieron en 2011 por la falta de agua y en el marco de un conflicto entre dos Municipios debido a un proyecto de acueducto encarado por el gobierno provincial. En febrero de 2012, se realizó un encuentro intercooperativo en el cual sobresalió la importancia de encarar políticas regionales.

Por su parte, en una entrevista concedida a una radio local a principios del año 2012, un intendente afirmaba que "el tema de la emergencia hídrica está en el marco de una política hídrica regional que hay que mirar realmente con ojos y decisiones políticas, que algunas ya se empezaron a mirar desde el año pasado, y que tienen que ver con un proyecto sustentable a mediano y largo plazo".

La región, de esta manera, forma parte de un lenguaje sobre el espacio-territorio en relación a la crisis hídrica. Como se puede apreciar, la política hídrica no se reduce a niveles de localidad o a unidades administrativas locales, sino que las excede y las conecta. Sin embargo, la región no expresa aquí un espacio delimitado, sino mas bien un tipo de acción y una forma de crear relaciones entre instituciones y actores. Entonces, su anclaje espacial es diferente en comparación con las cuestiones que remiten a las reservas. Los sentidos de la región están aquí asociados, por los actores, con relaciones cooperación interinstitucionales, con la negociación conjunta entre cooperativas $\mathrm{y} / \mathrm{o}$ municipios para 
realizar reclamos al gobierno provincial, para definir líneas y programas de acción, para reconocer problemas comunes, etc.

Si bien podría decirse que 'lo regional' refiere aquí a una suerte de anclaje espacial mas bien lábil si lo comparamos con las lógicas jurisdiccionales de las reservas -debido a que no implica ordenamientos jurídicos ni el establecimiento de límites, tampoco prohibiciones o regulaciones específicas-, esto no significa que la región esté al margen de la producción del espacio-territorio. El ordenamiento territorial y la región remiten a formas diferenciables, aunque complementarias, por ser parte de un mismo proceso general de producción del espacio-territorio.

A la dinámica de las reservas que hemos descrito subyace un conflicto mucho más amplio relativo al control, la apropiación y el uso del territorio en relación al recurso hídrico. Las reservas tienen toda su historia propia, que se explica en términos de negociaciones y conflictos que tienen una impronta específicamente local. En ciertos lugares, reservas municipales pueden lindar con reservas provinciales, pero sus respectivos procesos de creación y gestión son diferentes e independientes entre sí. Uno de los efectos planificados de la creación de relaciones regionales que ponderan lo supra-local fue la revitalización de un proyecto de larga data que plantea la creación de un cordón de reservas hídricas interconectadas a lo largo de toda la sierra, bajo el cual las diferentes reservas existentes, así como las proyectadas, comiencen paulatinamente a subsumirse.

La iniciativa de crear un cordón de reservas puede entenderse como una dinámica de relativización de una serie de territorios absolutos. En términos absolutos, cada reserva es un mundo en sí mismo que se puede describir aisladamente a partir de una serie de atributos y procesos que la objetivan como un territorio delimitado, diferenciable. De esta manera, el territorio se nos presenta como discreto, como aislado de otros. Pero al conectar a estas reservas distintas y asiladas en un conjunto mayor, el territorio se visibiliza como producto de relaciones sociales específicas.

Asimismo, en los últimos dos años fue impulsada la creación de una Coordinadora Ambiental y Derechos Humanos Sierras Chicas que reúne a organizaciones ambientalistas de todas las localidades de las sierras. Generar iniciativas regionales se nos presenta entonces tanto como un proceso de creación de relaciones sociales, como una dimensión de la producción social del espacioterritorio. Como un proceso, en definitiva, que produce su propio marco espacial.

\section{PALABRAS FINALES}

Debido a que el agua es un recurso natural, el problema del espacio-territorio es central en la cuestión hídrica. Como decíamos, las acciones en torno a la crisis hídrica en la zona inciden sobre el espacio-territorio, y en muchos casos la organización territorial establecida es objeto de intervención social y política planificada. No hay mejor ejemplo que los conflictos en torno a los usos y manejos de las reservas hídricas, de los cuales hemos mencionado sólo algunos. El espacio-territorio en su forma relacional está incrustado o internalizado en los procesos sociales. Así, "un evento o una cosa en un punto del espacio no puede ser entendida apelando sólo a lo que existe en ese punto. Depende de todo lo que está sucediendo alrededor". (HARVEY, 2006, p. 274) Depende, también, de las otras formas en que se manifiesta.

Las tres formas espaciales (absoluta relativa y relacional) no son excluyentes. Las reservas, como unidades territoriales cuyos límites están jurídicamente fijados, se nos presentan como espacios absolutos; es decir, como territorios. Pero se nos manifiestan como relativos si incorporamos la posición de la mirada en el análisis, y asimismo definidos relacionalmente en función de los conflictos que llevaron a su creación y los que conllevan una vez creadas y consideradas como espacios absolutos. El espacio relacional es, justamente, el espacio entendido en términos socio-políticos. Pero las reservas pueden aparecer también como territorios relativos, como sucedió para el caso del trazado de la calle en la Comuna y para la ruta E57.

Esta visión dinámica del espacio nos obliga a considerar que, en sí mismo, éste no es ni absoluto, ni relativo ni relacional, tal como afirma Harvey. Puede adoptar cualquiera de las formas, dependiendo de las circunstancias y de los proyectos socio-políticos en juego. Esto es incluso aún más crucial cuando las formas absolutas operan como constreñimiento y freno para formas que se proponen como alternativas, o cuando las formas 
establecidas pretenden ser modificadas. Cabría pensar, en este sentido, si los conflictos ambientales en general pueden ser comprendidos en términos de la tensión entre estas tres formas que adopta el espacio-territorio. En este sentido, todo proyecto ambiental es un proyecto socio-político, pues se 'enfrenta' a formas establecidas de organización espacio-territorial; es decir, a las formas absolutas. Pero debe lidiar asimismo con las formas relativas, que implican diferentes posicionamientos no sólo en torno a la 'mirada' sobre él, sino a las maneras diferenciales de producirlo, y que están en tensión con todas las otras maneras posibles.

\section{REFERENCIAS}

ABÉLÈS, M. Identity and Borders: An Anthropological Approach to EU Institutions. Twenty First Century Papers: On Line Working Papers from the The Center for 21st Century Studies University of Wisconsin. Milwaukee, n. 4, 2004. Disponible en: $<$ http://www4. uwm.edu/c21/pdfs/workingpapers/abeles.pdf>. Acceso: 15 de diciembre de 2013.

BALBI, F.; ROSATO A.; BOIVIN, M. La integración como representación social: eventos ritualizados en el proceso de integración. En: ROSATO, Ana (comp.). Construyendo integración al interior del Mercosur: la integración entrerriano-riograndense (1992-2001). Serie 'Antropología Política y Económica' - GIAPER. Buenos Aires: Antropofagia, 2009. p. 81-106.

BALBI, F.; A. R. Introducción. En: ROSATO, Ana y Fernando BALBI. (eds.) Representaciones sociales y procesos políticos. Estudios desde la Antropología Social. Buenos Aires: Antropofagia. 2003. p.

BARTH, F. O guru, o iniciador e outras variações antropológicas. Rio de Janeiro: Contra Capa Livraria. 2000. p. 243.

BRIONES, C. (comp.). Cartografias Argentinas: politicas indígenas y formaciones provinciales de alteridad. Buenos Aires: Antropofagia. 2005. p. 317.

CHAPMAN, Malcom. The commercial realization of the community boundary, en: GODDARD, Victoria;
LLOBERA, Joseph y SHORE, CHRIS (eds.): The anthropology of Europe. Identities and boundaries in conflict. Oxford; Providence: Berg, 1994. p. 310.

EVANS-PRITCHARD, E. E. Los Nuer. Barcelona: Anagrama. 1997. p. 284.

FRIEDMAN, J. 1994: Cultural identity \& global process. London, Sage Publications.

GAZTAÑAGA, Julieta. Un nuevo bloque político y económico: análisis antropológico del proceso político tejido en torno a la construcción de la Región Centro. Buenos Aires: Antropofagia. 2008. p. 141.

HARVEY, D. Justice, Nature \& the Geography of Difference. Oxford: Blackwell. 2004. p. 486.

HARVEY, D. Space as a key word. En: CASTREE, Noel; DERECK, Gregory. David Harvey. A critical reader. Oxford: Blackwell. 2006. p. 324.

HARVEY, D. Espacios de esperanza. Madrid: Akal. 2007. p. 328.

HARVEY, D. Espacios del capital. Hacia una geografia crítica: Akal. 2009. p. 445.

HEREDIA, B. Acerca del concepto de región. Estudios, n. 11-12, Centro de Estudios Avanzados de la Universidad de Córdoba, p. 83-97, enero / diciembre 1999.

KOBERWEIN, A. 2013. La producción de jerarquías espacio-territoriales en la gestión de recursos hídricos. En: Actas de las VII Jornadas de Investigación en Antropología Social. Buenos Aires. FFyL - UBA.

LEFEBVRE, H. La producción del espacio. En: Papers. Revista de sociología. Universidad Autónoma de Barcelona, v3, p. 219-229, 1974. Disponible en: $<$ http://papers.uab.cat/article/download/v3-lefebvre/ pdf-es> Acceso: 12 de abril de 2012.

LITTLE, P. Superimposed cosmographies on regional amazonian frontiers. Série Antropologia, Brasilia, n. 
219, 1997. Disponible en:

$<$ http://www.dan.unb.br/images/doc/Serie219empdf. pdf> Acceso: 28 de marzo de 2013.

MARX, C. El Capital. Crítica de la economía política. Tomo II. México: Fondo de Cultura Económica. 1994 [1867]. p. 623.

PACHECO DE OLIVEIRA, J. Una etnografía de las tierras indígenas: procedimientos administrativos y procesos políticos. En: PACHECO DE OLIVEIRA, JOÃO (comp.) Hacia una antropología del indigenismo. Rio de Janeiro / Lima: Contracapa. 2006. p.228.

PITT-RIVERS, J. Un pueblo de la sierra: Grazalema. Alianza: Barcelona. 1994. p.272.

RANKLIN, K. Anthropologies and geographies of globalization. Progress in Human Geography, London, v. 27, n. 6, p. 708-734, 2003. DOI: $10.1191 / 0309132503$ ph457oa

ROSATO, A. El proceso de institucionalización de la integración. En: ROSATO, Ana (comp.) Construyendo integración al interior del Mercosur: la integración entrerriano-riograndense (1992-2001). Serie Antropología Política y Económica - GIAPER. Buenos Aires: Antropofagia. 2009. p. 53-80.

SANTOS, M. Por una geografía nueva. Madrid: Espasa Calpe. p. 257.

SHORE, C. Building Europe. The cultural politics of European Union. London: Routledge. 2000. p. 258.

SIMMEL, G. El Espacio y la Sociedad. En: Sociología. Estudios sobre las formas de socialización. Buenos Aires: Espasa-Calpe. 1939. pp. 207-296.

THER RÍOS, F. Complejidad territorial y sustentabilidad: notas para una epistemología de los estudios territoriales. Horizontes Antropológicos, Porto Alegre, v. 12, n.25, p. 105-115, enero / junio 2006. DOI: http://dx.doi.org/10.1590/S010471832006000100006 